\title{
Bipolar chaotic pulse position modulation communication system based on cyclic LDPC
}

\author{
Hui Li ${ }^{1 *}$, Hanyu Liu ${ }^{1 *}$ and Sina Vafi ${ }^{2}$
}

\begin{abstract}
To overcome the error propagation and improve the communication efficiency of the chaotic pulse position modulation (CPPM) system, the bipolar chaotic pulse position modulation (BCPPM) communication system is proposed here. In BCPPM, every two-bit data are set as a group. The first bit and the discrete chaotic map determine the position of sent pulse, while the second bit determines the polarity of sent pulse. Each pulse in the channel contains two-bit information, so this scheme improves the communication efficiency. A $(255,175)$ cyclic low-density parity-check code (LDPC) was designed, and its generator matrix and parity check matrix are cycled. Furthermore, the constructed BCPPM communication system is utilized in the cyclic LDPC aiming to mitigate the effect of noise. In the transmitter, it uses the encoder structure of cyclic codes while the min-sum algorithm is deployed to decode in the receiver. The analysis indicates that the proposed system is secure, insensitive to the channel distortion and convenient for multiple access communication. The simulation results show that in the additive white Gaussian noise (AWGN) channel, multipath channel, multiuser model, and hybrid communication environment, the BCPPM system has lower bit error rate (BER) compared with those of the CPPM and chaotic pulse on-off-keying (CPOOK). In addition, using cyclic LDPC codes, the system is more suitable for hardware implementation.
\end{abstract}

Keywords: Chaotic pulse position modulation; Multipath channel; Multiuser; Cyclic-LDPC

\section{Introduction}

The ultra wideband (UWB) communication system is widely used in communication systems especially in military applications. This technique not only meets the requirements of traditional communication system, but also provides a secure transmission. Due to the flexible working environment, such as indoor or outdoor, it can overcome the multipath propagation and interference from other devices effectively $[1,2]$.

Chaotic communication offers high security and a low probability of intercept [3]. However, since the chaotic synchronization is very sensitive to the signal distortion, filtering and channel noise, utilizing the chaotic communication systems encounters many difficulties in practical applications [4]. In view of the good robust performance of UWB communication on filtering and channel noise, some scholars combined the UWB with chaotic communication and proposed a model of communication system,

\footnotetext{
* Correspondence: li20042007@163.com; hanyuliu@live.cn

${ }^{1}$ School of Electrical Engineering and Automation, Henan Polytechnic

University, Jiaozuo 454000, China

Full list of author information is available at the end of the article
}

named as chaotic pulse position modulation (CPPM) [5]. In the CPPM system, the intervals between pulses are chaotically changed based on the chaotic map. This communication scheme protects information from interception. Besides, the status information of the chaotic system is completely contained in the time intervals of the pulses with the same shape. The distortion caused by filter and channel will only destroy the shape of pulses and will not affect the intervals between them. In this case, the CPPM system is insensitive to the multipath channel distortion [6]. This makes the chaotic pulse signal much easier to be reused and achieve multiple access communication than continuous chaotic signal [7].

Although CPPM has many advantages, it induces additional delay into the chaotic map that not only causes the chaotic map to diverge easily, but also leads to error propagation. A method was proposed as the chaotic pulse on-off keying (CPOOK) [8], which does not have any feedback loop in its structure. Therefore, it has lower bit error rate (BER) than the CPPM system in the 
case of non-ideal timing. But each bit signal needs to transmit extra synchronization pulse in this scheme, which reduces the efficiency of the transmitter. A method of MCPPM was proposed in [9], where the delay block, which is decided by the data, is sent outside the feedback loop. In this way, the communication system overcomes the error propagation phenomenon. In order to further improve the efficiency of communication, a bipolar chaotic pulse position modulation (BCPPM) communication system was put forward in this paper. It sends two-bit data in each pulse. While retaining the advantages of the CPPM system, such as high safety, insensitivity to the channel distortion, and simplicity for multiple-access communication, the BCPPM system has higher communication efficiency and lower BER.

The low-density parity-check code (LDPC) [10] demonstrates a good performance close to the Shannon limit and also low decoding complexity and flexible structure. These factors make it an appropriate option in the research field of channel coding $[11,12]$. Cyclic codes are an important subclass of linear codes, which have the general properties of linear codes and also the circulation. The cyclic shift of any code word in the code group is still a code word in this code group [13]. The characteristic of circulation is such that its encoding and concomitant formula can be achieved by a feedback shift register circuit, which is easily implemented on hardware. With the characteristics of cyclic codes and LDPC codes, the generator matrix and parity-check matrix of the cyclic LDPC code are cyclic, so the encoder can be realized by the feedback shift register to improve the speed of hardware system. A $(255,175)$ cyclic-LDPC code designed, and it is used for the BCPPM system to reduce the system BER.

\section{Bipolar chaotic pulse position modulation communication system}

\subsection{CPRG and CPPM}

In this section, we describe the BCPPM communication system. To begin with, we introduce the chaos pulse regenerator (CPRG). The block diagram of the CPRG is presented in Figure 1. When the value of time $T$ reaches the value of delay $T_{n}$ for the $n$th round, the comparator will send an enabled signal to trigger:

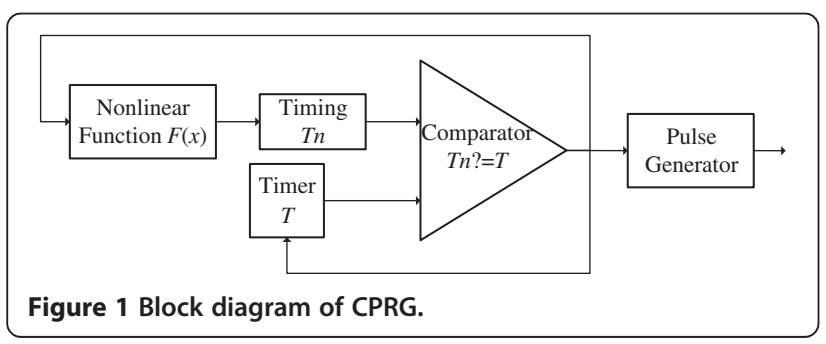

a) The function $F(x)$ calculates the delay value $T_{n+1}$ for the next round;

b) The timer resets and starts timing for the next round;

c) The pulse generator outputs a narrow pulse whose width is $T_{w} \ll T_{n}(n=1,2, \ldots)$.

The sequence of time intervals $\left\{T_{n}\right\}$ represents the iterations of a chaotic process. It can be calculated by the function $F(x)$ which is always a nonlinear function, $T_{n}=$ $F\left(T_{n-1}, T_{n-2}, \ldots, T_{1}\right)$. From the formula, we know that $T_{n}$ is determined by the former $n-1$ delay value. When $n=1$, it is just a function of one variable. Through the $F(x)$, the output of CPRG feedbacks to itself directly, constituting a feedback loop. Some studies on such CPRG can be found in $[9,10]$.

In CPPM, the information is encoded within the chaotic pulse signal by using additional delays inside the feedback loop, in other words, by changing the intervals $T_{n}$. When transmitting ' 0 ', the CPRG will output the pulse immediately, while transmitting ' 1 ', the pulse will delay for a fixed time $T_{d} T_{d} \ll T_{n}(n=1,2, \ldots)$. At this time, the $F(x)$ function can be expressed as $T_{n}=F\left(T_{n-1}\right.$, $\left.T_{n-2}, \ldots, T_{1}, T_{d}\right)$. And in this way, the CPRG output waveform contains the binary information. The block diagram of classical CPPM is shown in Figure 2.

\subsection{BCPPM modulator}

BCPPM is based on the CPRG, and the modulator of BCPPM is shown in Figure 3. When the system initialization is complete, CPRG unit starts working: The nonlinear function outputs the delay time value $T_{n}$; the timer starts timing; the comparator compares its two inputs constantly. When CPRG outputs the enable signal, the system reads the first bit data to decide whether to add the $T_{d}$ before entering later modules. After that, it reads the second bit data to choose the polarity of the narrow pulse for output.

In Figure 3, we can see that the sent data are grouped in every two bits. In each round, the first bit data and the chaotic map determine the position of pulse and the second bit determines its polarity. The delay module is moved outside of the feedback loop compared with that of CPPM. It makes the signal intervals of CPRG only relevant to the states of nonlinear function which is inside the CPRG. They are independent of the information sequence. Here, we get $T_{n}=F\left(T_{n-1}\right)$. In this way, it avoids the possible adverse effects of information sequence to the chaotic map and keeps the no regularity of output pulse intervals sequence of CPRG. Besides, it eliminates the possibility of divergence. In each transmission period, the BCPPM modulator outputs a bipolar pulse, which contains two-bit information under the same conditions with CPPM. So, the BCPPM scheme doubles the information rate. 


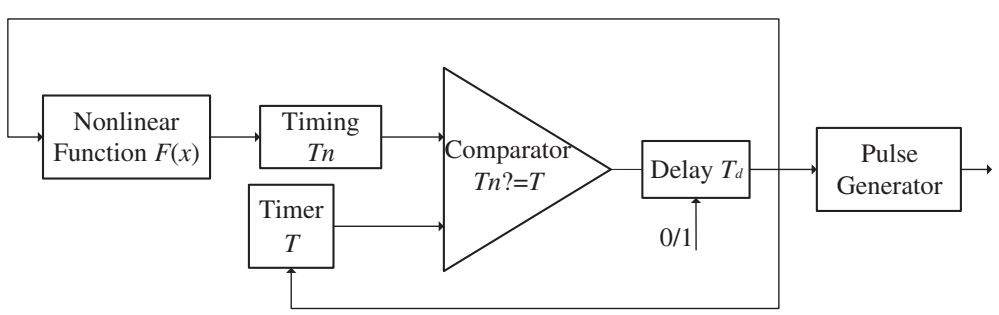

Figure 2 Block diagram of classical CPPM.

Through the above analysis, the transmitted signal of BCPPM can be expressed as

$$
U(t)=\sum_{j=0}^{\infty}(-1)^{b_{2 j+1}} \omega\left(t-t_{j}\right)
$$

where $\omega(t)$ is the baseband transmission signal.

$$
t_{j}=t_{0}+\sum_{j=0}^{j-1} T_{n}+b_{2 j} T_{d}
$$

where $t_{0}$ is the initial delay for system.

$b_{j}$ is the data to be transmitted:

$$
b_{j}= \begin{cases}0 & j=1,2, \cdots \\ 1 & \end{cases}
$$

\subsection{BCPPM demodulator}

Pulse sequence with delay and polarity modulation becomes the signal to be transmitted in the channel. If an unauthorized receiver does not know the interval information of pulse sequence, it is impossible to judge whether a received pulse was delayed. Therefore, it cannot judge whether ' 0 ' or ' 1 ' is transmitted at this moment. If there is an ideal synchronization between the transmitter's CPRG and the receiver's CPRG, it will output the same signal with the sent signal in channel except some of the pulses delayed due to the data modulation. By estimating the received signal and the time when CPRG outputted the corresponding pulse, the first bit data can be restored. On this basis, according to the polarity information of pulse, the second bit data can be judged. While the CPRGs of both sides of the transceiver are not enough to match, it will generate a large number of errors. Therefore, the initial parameters of CPRG act like a private key and play a decisive role in the transmission.

In the case of ideal synchronization between transmitter and receiver, the receiver will know the time or time window when the pulse is expected to appear. That allows the output of the receiver to be kept locked before the appearance of excepted pulses. During this period, other users can transmit their information pulses. In $\mathrm{BCPPM}$, there are two time windows in the receiver. The BCPPM receiver is shown in Figure 4. Based on the synchronization of the transmitter and the receiver, the CPRG of the receiver enables the sampler near the time when the pulse is expected to appear. It will create a time window which contains the expected pulse, then divide the window into two parts based on the time $T_{d}$, time window ' 0 ' and time window ' 1 ', respectively. The signal in time window ' 0 ' and time window ' 1 ' are transferred to a two-peak detector, then it figures out the two bits of information. The detailed demodulation process can be described as follows:

While demodulating the two-bit data, priority should be given to the first bit, then the second one. The first bit, delay information, can be judged from the sampling value in which window it has the larger peak. If the peak

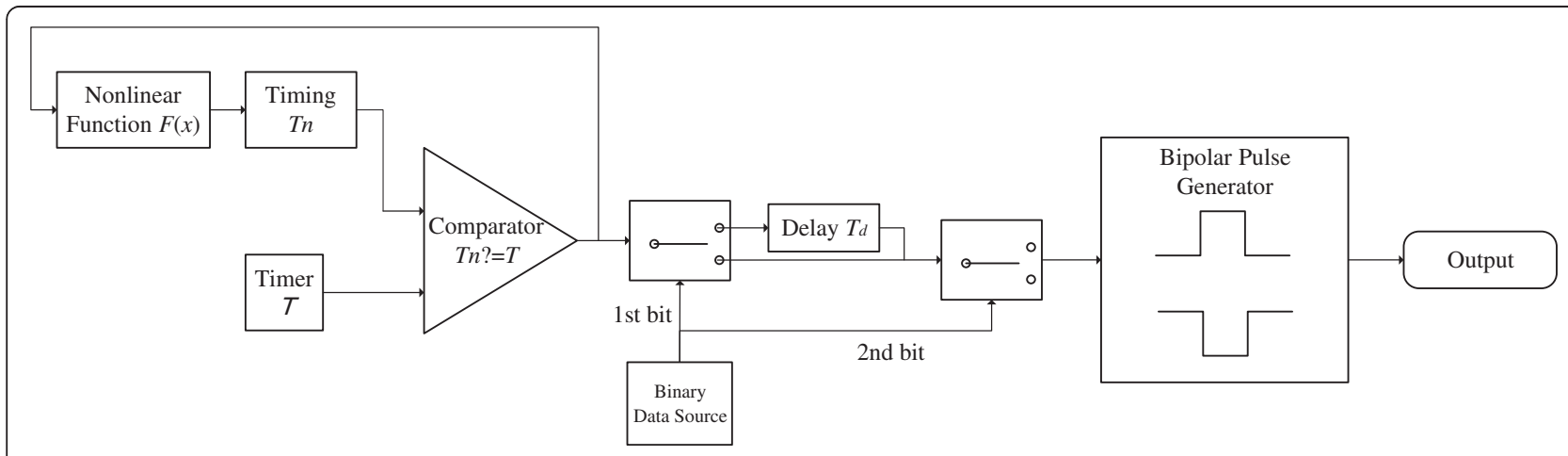

Figure 3 Block diagram of BCPPM modulator. 


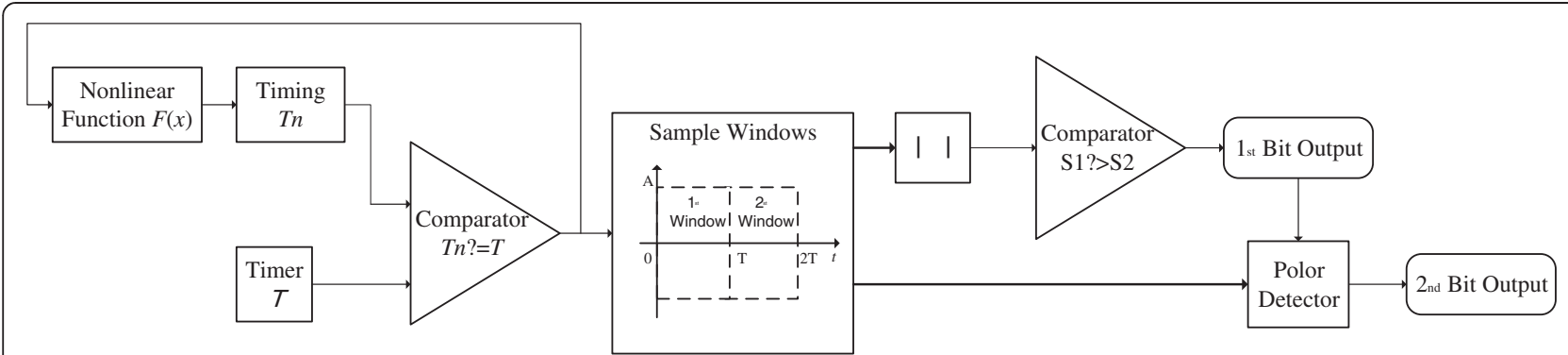

Figure 4 Block diagram of BCPPM demodulator.

is in window ' 0 ', the first bit is ' 0 '. Otherwise, it is ' 1 '. On this basis, the detector detecting the polarity of pulse in the above time window, if it is positive, the second bit information is ' 0 '. Otherwise, it is ' 1 '. So, there are a total of four cases for the two-bit data, as it is shown in Figure 5.

It is clear that the feedback loop in CPPM was removed in Figure 4. This eliminates the dependence of receiver CPRG synchronization on the accuracy of the demodulator judgment. It also eliminates the asynchronous problem between transmitter's CPRG and receiver's CPRG due to the wrong judgments, thus avoiding the error propagation. Thus, the BCPPM scheme greatly improves the practicability of the system.

\subsection{Performance of BCPPM}

Let $x_{1}=1$, denoting that the demodulation of the first bit is incorrect, and $x_{1}=0$, denoting that the demodulation of the first bit is correct. With the same notation, $x_{2}=1$, which means that the demodulation of the second bit is incorrect; while $x_{2}=0$, which means that the demodulation of the second bit is correct. The first bit and the second bit are dependent in the demodulator of the BCPPM system. The judgment of the second bit depends on the time window which is decided by the first
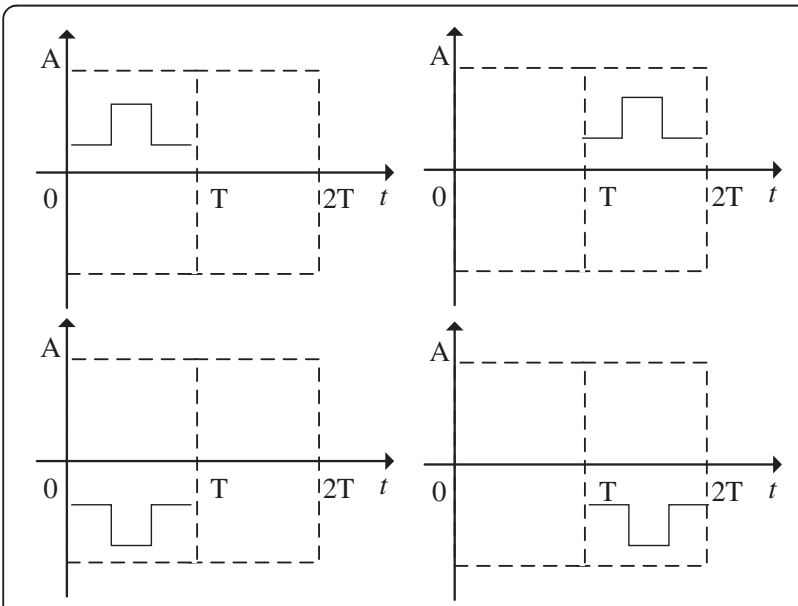

Figure 5 Cases of the BCPPM receiver. bit. So the output of the first bit will affect the judgment of the second bit. If the demodulation of first bit is incorrect, as a result, the second bit will not fall in the corresponding time window. The sampling value of the signal is the noise level so the judgment is like coin tossing. In this case, the probability of correct demodulation for the second bit is 0.5 . So, in the case of ideal timing, the BER of the BCPPM system is the following:

$$
\begin{aligned}
P_{e}= & \frac{1}{2}\left(p\left(x_{1}=1, x_{2}=0\right)+p\left(x_{1}=0, x_{2}=1\right)+2\right. \\
& \left.\times p\left(x_{1}=1, x_{2}=1\right)\right) \\
=\frac{1}{2} & \left(p\left(x_{1}=1\right) \times p\left(x_{2}=0 \mid x_{2}=1\right)+p\left(x_{1}=0\right)\right. \\
& \times p\left(x_{2}=1 \mid x_{1}=0\right)+2 \times p\left(x_{1}=1\right) \\
& \left.\times p\left(x_{2}=1 \mid x_{1}=1\right)\right) \\
=\frac{1}{2} & \left(p\left(x_{1}=1\right) \times \frac{1}{2}+p\left(x_{1}=0\right) \times p\left(x_{2}=1 \mid x_{1}=0\right)\right. \\
& \left.+2 \times p\left(x_{1}=1\right) \times \frac{1}{2}\right) \\
=\frac{1}{2} & \left(Q\left(\sqrt{\frac{E_{b}}{N_{0}}}\right) \times \frac{1}{2}+\left(1-Q\left(\sqrt{\frac{E_{b}}{N_{0}}}\right)\right)\right. \\
& \left.\times Q\left(\sqrt{\frac{2 E_{b}}{N_{0}}}\right)+Q\left(\sqrt{\frac{E_{b}}{N_{0}}}\right)\right) \\
=\frac{3}{4} & \left(Q\left(\sqrt{\frac{E_{b}}{N_{0}}}\right)\right)+\frac{1}{2}\left(1-Q\left(\sqrt{\frac{E_{b}}{N_{0}}}\right)\right) \times Q\left(\sqrt{\frac{2 E_{b}}{N_{0}}}\right)
\end{aligned}
$$

\section{Cyclic LDPC codes of system \\ 3.1 Cyclic LDPC codes}

Cyclic codes and LDPC codes are all linear block codes, and some special cyclic codes are LDPC codes at the same time. This is the so-called cyclic LDPC. For example, a generator polynomial of $(15,7)$ cyclic LDPC is: $g(x)=x^{8}+x^{7}+x^{6}+x^{4}+1$. According to the generator polynomial, we get the generator matrix $G$ and check matrix $H$ as follows: 


$$
\begin{aligned}
G=\left(\begin{array}{lllllllllllllll}
1 & 0 & 0 & 0 & 1 & 0 & 1 & 1 & 1 & 0 & 0 & 0 & 0 & 0 & 0 \\
0 & 1 & 0 & 0 & 0 & 1 & 0 & 1 & 1 & 1 & 0 & 0 & 0 & 0 & 0 \\
0 & 0 & 1 & 0 & 0 & 0 & 1 & 0 & 1 & 1 & 1 & 0 & 0 & 0 & 0 \\
0 & 0 & 0 & 1 & 0 & 0 & 0 & 1 & 0 & 1 & 1 & 1 & 0 & 0 & 0 \\
0 & 0 & 0 & 0 & 1 & 0 & 0 & 0 & 1 & 0 & 1 & 1 & 1 & 0 & 0 \\
0 & 0 & 0 & 0 & 0 & 1 & 0 & 0 & 0 & 1 & 0 & 1 & 1 & 1 & 0 \\
0 & 0 & 0 & 0 & 0 & 0 & 1 & 0 & 0 & 0 & 1 & 0 & 1 & 1 & 1
\end{array}\right) \\
H=\left(\begin{array}{llllllllllllllll}
1 & 1 & 0 & 1 & 0 & 0 & 0 & 1 & 0 & 0 & 0 & 0 & 0 & 0 & 0 \\
0 & 1 & 1 & 0 & 1 & 0 & 0 & 0 & 1 & 0 & 0 & 0 & 0 & 0 & 0 \\
0 & 0 & 1 & 1 & 0 & 1 & 0 & 0 & 0 & 1 & 0 & 0 & 0 & 0 & 0 \\
0 & 0 & 0 & 1 & 1 & 0 & 1 & 0 & 0 & 0 & 1 & 0 & 0 & 0 & 0 \\
0 & 0 & 0 & 0 & 1 & 1 & 0 & 1 & 0 & 0 & 0 & 1 & 0 & 0 & 0 \\
0 & 0 & 0 & 0 & 0 & 1 & 1 & 0 & 1 & 0 & 0 & 0 & 1 & 0 & 0 \\
0 & 0 & 0 & 0 & 0 & 0 & 1 & 1 & 0 & 1 & 0 & 0 & 0 & 1 & 0 \\
0 & 0 & 0 & 0 & 0 & 0 & 0 & 1 & 1 & 0 & 1 & 0 & 0 & 0 & 1
\end{array}\right)
\end{aligned}
$$

Having the $G$ and $H$ matrices we can see that this cyclic code is also a LDPC code and irregular one.

In this paper, we design a $(255,175)$ cyclic LDPC, whose generator polynomial is as follows:

$$
\begin{aligned}
& g(x)=x^{80}+x^{78}+x^{76}+x^{74}+x^{71}+x^{69}+x^{68}+x^{67} \\
& \quad+x^{65}+x^{64}+x^{62}+x^{60} \\
& +x^{59}+x^{56}+x^{55}+x^{52}+x^{50}+x^{48}+x^{46}+x^{43}+x^{41} \\
& +x^{40}+x^{39}+x^{38} \\
& +x^{37}+x^{28}+x^{27}+x^{26}+x^{24}+x^{23}+x^{22}+x^{20}+x^{19} \\
& +x^{18}+x^{17}+x^{16} \\
& +x^{15}+x^{14}+x^{12}+x^{11}+x^{10}+x^{8}+x^{7}+x^{4}+1
\end{aligned}
$$

Since the $G$ matrix satisfies the cyclic characteristics, we can use the feedback shift register to encode the $(255,175)$ cyclic LDPC code at the transmitter, and use the LDPC decoding algorithm at the receiver. Thus, the design combines the hardware (such as FPGA) easy to implement of cyclic codes and low decode BER of LDPC codes. It can obtain high communication quality.

\subsection{LDPC codes decoding algorithm}

The Min-sum decoding algorithm is a simplified approximate algorithm of the log domain belief propagation (BP) decoding algorithm [14]. It uses minimum operation to simplify the function operation and to reduce the computational complexity greatly. What is more, it does not need to estimate the channel noisy and has no complex operations. So it is easy for hardware implementation. But its performance is reduced when compared with the BP decoding algorithm [15]. In general, the Min-sum decoding algorithm is very suitable to the occasion where one has limited hardware conditions. Here, the Min-sum decoding algorithm is used for the BCPPM communication system.

The Min-sum algorithm is as follows:

a) Initialization

$$
L\left(q_{i, j}\right)=L\left(P_{i}\right)
$$

b) Check to bit

$$
L\left(r_{j i}\right)=\prod_{i^{\prime} \in R_{j} i} \operatorname{sign}\left(L\left(q_{i^{\prime} j}\right)\right) \times \min _{i^{\prime} \in R_{j} i}\left(\left|L\left(q_{i^{\prime} j}\right)\right|\right)
$$

c) Code word test

$$
\begin{aligned}
& L\left(q_{i}\right)=L\left(P_{i}\right)+\sum_{j \in C_{i}} L\left(r_{j i}\right) \\
& L\left(q_{i}\right)>0, \quad \hat{c}_{i}=0 \\
& L\left(q_{i}\right) \leq 0, \quad \hat{c}_{i}=1
\end{aligned}
$$

Here, if $H c^{T}=0$ or the iteration count is equal to the maximum number of iterations, system will output the decoding results.

d) bit to Check

$$
L\left(q_{i j}\right)=L\left(P_{i}\right)+\sum_{j^{\prime} \in C_{i j}} L\left(r_{j^{\prime} i}\right)
$$

Then, return to step $b$. 


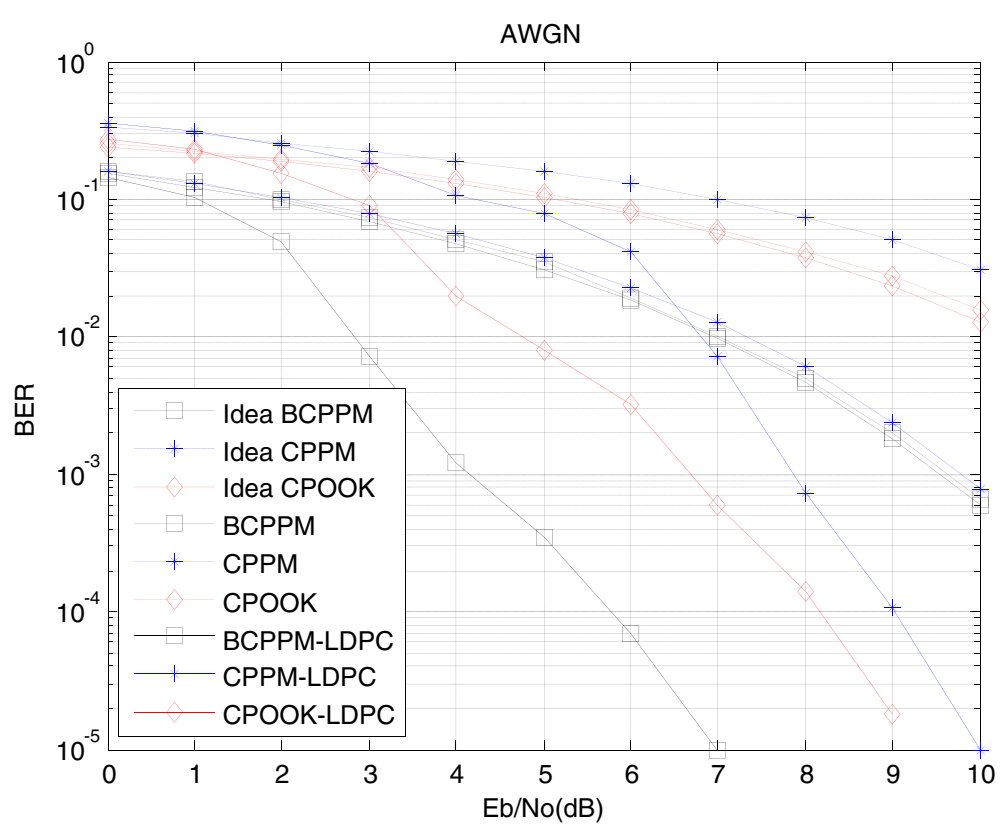

Figure 6 Simulation of additive Gauss white noise channel model.

\section{Simulation and analysis}

4.1 Additive white Gaussian noise channel

Firstly, we use the additive white Gaussian noise (AWGN) channel model to simulate the BER of BCPPM, CPPM, and CPOOK. We consider the first and second bits together in the theoretical analysis and simulations, so the BER performance is the result of the whole BCPPM system. Simulation parameters are chosen as follows:
1) For simplicity, we use the one-dimensional logistic maps:

$$
T_{n}=a_{n} T_{n-1}\left(1-T_{n-1}\right)
$$

where $a_{n}=3.99, T_{0}=0.68$;

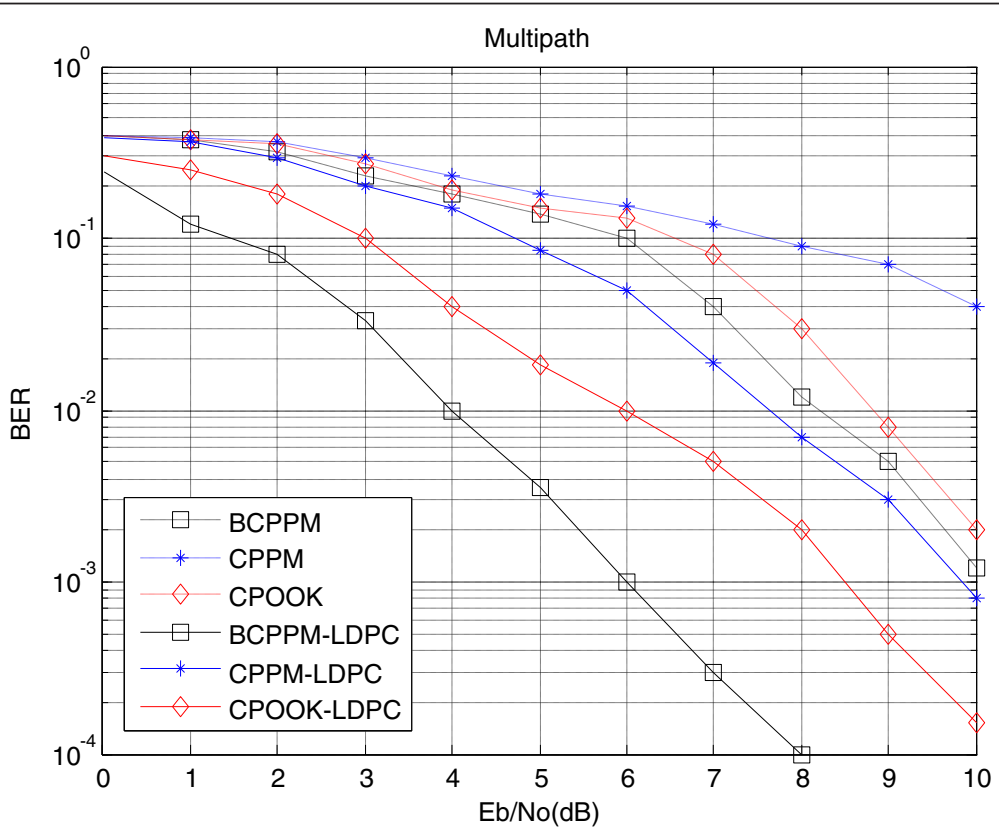

Figure 7 Simulation of multipath channel model. 


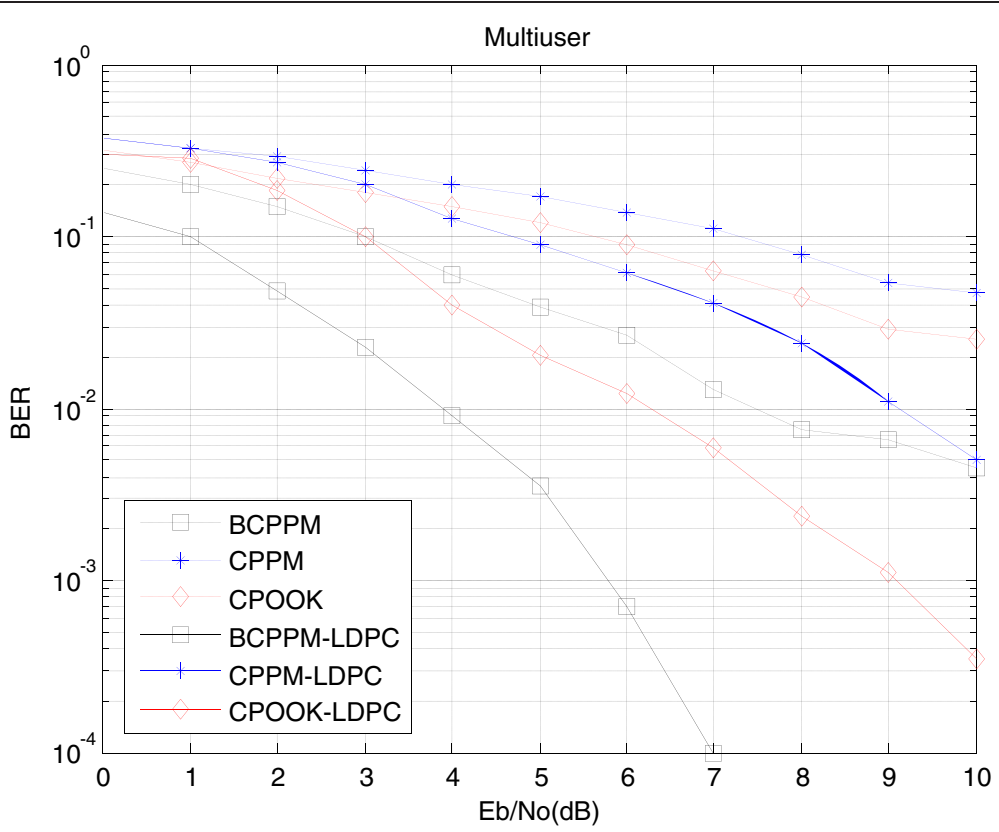

Figure 8 Simulation of multiple-access model.

2) The $(255,175)$ cyclic LDPC has been described in section 3.2. We can figure out the system rate is $\frac{35}{51}$ when using the LDPC code.

As shown in Figure 6, we get nine BER curves. They are the BCPPM, CPPM, and CPOOK systems in ideal condition; the BCPPM, CPPM, and CPOOK system simulations without LDPC; and the BCPPM, CPPM, and CPOOK simulations with LDPC. From the figure, we can see that the simulation results of $\mathrm{BCPPM}$ and $\mathrm{CPOOK}$ match the ideal curves well in the case of non-ideal timing because the structure of these systems can overcome the error propagation phenomenon. Furthermore, BCPPM has the lower BER compared with CPPM and CPOOK. Because each bit signal needs to transmit an extra synchronization pulse in CPOOK, the efficiency of the transmitter is

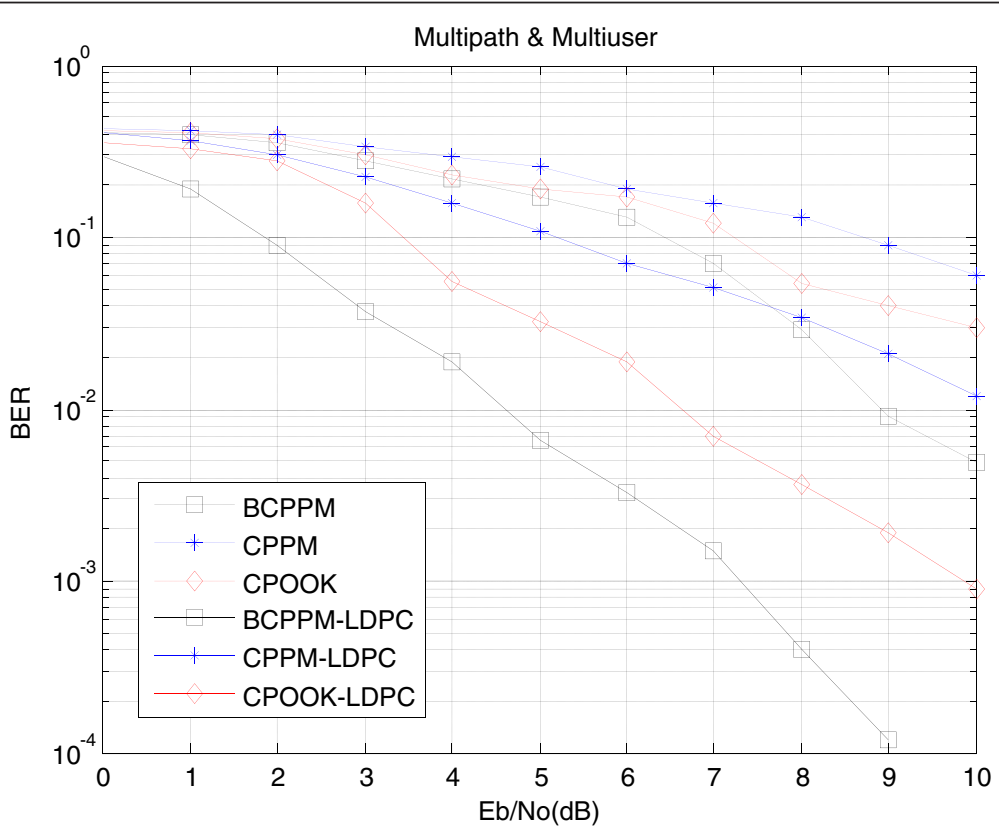

Figure 9 Simulation of multipath and multiple-access model. 
reduced. It is clear that the BER of all systems have greatly decreased after using cyclic LDPC.

\subsection{Multipath channel}

Here, we simulate the multipath channel model for BCPPM, CPPM, and CPOOK. For simplicity, the channel model is a two-path channel. The simulation parameters are chosen as follows:

1) Gain of the path 1 is $\alpha_{1}=0.8$ and path 2 is $\alpha_{2}=0.6$;

2) The path delays are $\tau_{1}=0$ and $\tau_{2}=2$ respectively;

3) The rest are same with section 4.1.

From the simulation results in Figure 7, we find that $\mathrm{BCPPM}$ has stronger multipath tolerance than CPPM and CPOOK. The reason is that the status information of chaotic system is completely contained in the time intervals of the pulses with the same shape. The distortion caused by the filter and the channel will only destroy the shape of pulses, and not affect the intervals between them.

\subsection{Multiuser model}

In BCPPM, CPPM, and CPOOK, multiuser communication means assigning different initial values to each user for the chaotic maps, as we have discussed in section 2.3. In this section, we simulate the multiuser model for $\mathrm{BCPPM}, \mathrm{CPPM}$, and CPOOK. For simplicity, there are two users in this model and the parameters are chosen as follows:

1) Initial value for the chaotic map of user 1 is $x_{0}^{(1)}=0.68$, the value of user 2 is $x_{0}^{(2)}=0.78$;

2) The rest are same with section 4.1.

The simulation results are shown in Figure 8. The figure shows that though the BCPPM system multiple access mode is simple, it still has low BER performance when using the LDPC codes. That is because the synchronization of the CPRG is between the transmitter and the receiver. Every user's receiver will be kept locked outside its time windows. If the pulses of different users are outputs in different time windows, there will be no multiuser interference from each other.

\subsection{Multipath channel and multiuser model}

Finally, we compare the performance of BCPPM, CPPM, and $\mathrm{CPOOK}$ in the multipath channel which has two users. The simulation parameters are same with those in section 4.2. and section 4.3 .

From the simulation results, we get four BER curves as before, as shown in Figure 9. It shows that, considering complex communications background of the multipath and multiuser, the BCPPM system still has a good communication performance.

\section{Conclusion}

In this paper, we proposed a BCPPM communication system, described the structure of transmitter and receiver, and then discussed the possibility of combining cyclic LDPC code with the system. The BCPPM system has the advantage of high security, insensitivity to channel distortion and simplicity of deploying in multiuser communication. The simulation results show that BCPPM system has a lower BER compared with the CPPM and CPOOK systems. Besides, the use of cyclic LDPC codes is more conductive for the hardware implementation and has better BER performance.

\section{Competing interests}

The authors declare that they have no competing interests.

\section{Acknowledgements}

This work was supported by the National Natural Science Foundation of China (Grant No. U1304523), the Young Scientists Fund of the National Natural Science Foundation of China (Grant No. 11105042) and the Henan Provincial Department of Education Science and Technology Research key project (No.13A510330).

\section{Author details}

${ }^{1}$ School of Electrical Engineering and Automation, Henan Polytechnic University, Jiaozuo 454000, China. ${ }^{2}$ School of Engineering and Information Technology, Charles Darwin University, Darwin 0909, Australia.

Received: 4 December 2013 Accepted: 12 June 2014

Published: 22 June 2014

\section{References}

1. YS Shen, FB Ueng, LD Jeng, A new time-hopping/direct-sequence biorthogonal PPM UWB communication system. EURASIP J. Wirel. Commun. Netw. 149, 1-11 (2011)

2. LC Tran, A Mertins, TA Wysocki, Unitary differential space-time-frequency codes for MB-OFDM UWB wireless communications. IEEE Trans. Wirel. Commun. 12(2), 862-876 (2013)

3. LS Jin, Y Zhang, LJ Li, One-to-many chaotic synchronization with application in wireless sensor network. IEEE Commun. Lett. 17(9), 1782-1785 (2013)

4. H Yang, GP Jiang, Reference-modulated DCSK: a novel chaotic communication scheme. IEEE Trans. Circuits Syst. II Expr. Briefs 60(4), 232-236 (2013)

5. M Sushchik, N Rulkov, L Larson, L Tsimring, H Abarbanel, K Yao, A Volkovskii, Chaotic pulse position modulation: a robust method of communication with chaos. IEEE Commun. Lett. 4(4), 128-130 (2000)

6. N Rulkov, M Sushchik, L Tsimring, A Volkovskii, Digital communication using chaotic-pulse-position modulation. IEEE Trans. Circuits Syst. 12(48), 1436-1444 (2001)

7. H Torikai, T Saito, W Schwarz, Multiplex communication scheme based on synchronization via multiplex pulse-trains, in Proceedings of the 1998 IEEE International Symposium on Circuits and Systems (Monterey, CA, USA, 1998), pp. 554-557

8. H Yang, GP Jiang, P Deng, Chaotic pulse On-off-keying modulation scheme for ultra-wide bandwidth communications. J. Electron. Inform. Technol. 29(3), 677-680 (2007)

9. $\quad \mathrm{H}$ Yang, GP Jiang, A modified, chaotic pulse position modulation scheme for ultra-wide bandwidth communication. J. Nanjing Univ. Posts Telecommun. 26(2), 47-50 (2006)

10. RG Gallager, Low-density parity-check codes. IRE Trans. Inform. Theor. 8(1), 21-28 (1962)

11. GH Zhang, R Sun, XM Wang, Several explicit constructions for (3, L) QC-LDPC codes with girth at least eight. IEEE Commun. Lett. 17(9), 1822-1825 (2013)

12. B Rong, YY Wu, G Gagnon, Multi-layer iterative LDPC decoding for broadband wireless access networks: a recursive shortening algorithm. IEEE Trans. Wirel. Commun. 12(3), 1320-1327 (2013) 
13. SX Li, SH Hu, T Feng, GN Ge, The weight distribution of a class of cyclic codes related to Hermitian forms graphs. IEEE Trans. Inf. Theory 59(5), 3064-3067 (2013)

14. DJC Mackay, Good error-correcting codes based on very spares matrices. IEEE Trans. Inf. Theor. 45, 399-431 (1999)

15. EB Li, D Declercq, K Gunnam, Trellis-based extended min-sum algorithm for non-binary LDPC codes and its hardware structure. IEEE Trans. Commun. 61(7), 2600-2611 (2013)

doi:10.1186/1687-1499-2014-105

Cite this article as: Li et al:: Bipolar chaotic pulse position modulation communication system based on cyclic LDPC. EURASIP Journal on Wireless Communications and Networking 2014 2014:105.

Submit your manuscript to a SpringerOpen ${ }^{\odot}$ journal and benefit from:

- Convenient online submission

- Rigorous peer review

- Immediate publication on acceptance

- Open access: articles freely available online

- High visibility within the field

- Retaining the copyright to your article 